\title{
A New Generation of Translational Tools designed to Monitor Multiple Sclerosis (MS) at Clinical and Subclinical Stages
}

\author{
Studneva $\mathrm{M}^{1}$, Suchkov $\mathrm{S}^{1-3 *}$, Rose $\mathrm{N}^{4}$, Gabibov $\mathrm{A}^{5}$ and Cheng $\mathrm{RH}^{6}$ \\ ${ }^{1}$ MINO MGUPP, Russia \\ ${ }^{2} \mathrm{AI}$ Evdokimov Moscow State Medical \& Dental University, Russia \\ ${ }^{3}$ EPMA, Belgium
}

${ }^{4}$ Johns Hopkins Center for Autoimmune Disease Research and PAHO/WHO Collaborating

Center for Autoimmune Disorders, Johns Hopkins Medical Institutions, USA, Harvard Medical

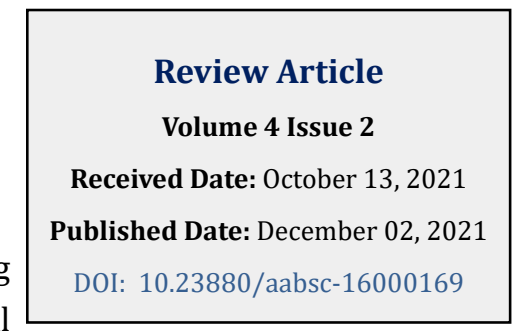

School, USA

${ }^{5}$ Institute for Bioorganic Chemistry, Russian Academy of Sciences, Russia

${ }^{6}$ Department of Molecular and Cellular Biology, UCDavis, USA

*Corresponding author: Sergey Suchkov, MINO MGUPP, Moscow, Russia, Email: ssuchkov57@gmail.com

\section{Abstract}

According to tremendous translational researchers, biomarkers as a part of the ligand-receptor tandems have induced an impulse to prompt the development of an upgraded concept of the targeted therapy. It is health indicators that justifying the necessity to create targeted drug of the next-step generation to be implemented at the clinical and sub-clinical key stages of the disease pathogenesis and to be involved into a multi-stage process to get the shifts appeared modified. Catalytic antibodies (CatAbs) have emerged as powerful tools for the efficient and specific catalysis of a wide range of chemical transformations. Generating antibody catalysts that achieve enzymatic efficiency remains a challenging task to be implemented in the designed translational applications. We also comment on recent developments in the screening CatAbs-related process that allow for a more efficient identification of Ab-based catalysts to be used further as native ones or engineered and/or designed bioproducts in clinical practice. The generation of an edited Abs, Eezyme or artificial ABZYME through transition state stabilization by Abs has been thus demonstrated. So, we may consider Ab-proteases as unique translational probes to diagnose, to monitor, to control and to treat and rehabilitate MS patients at clinical stages and to prevent the disorder at subclinical stages in personsat-risks to secure the efficacy of regenerative manipulations. There is an evident-based outcome of the latest studies in multiple sclerosis (MS) related fields that illustrates the proved targeted activity of Ab-proteases. High impact of Ab-proteases can be used to monitor both clinical and subclinical courses of chronic autoimmune inflammation (MS) to predict stepwise transformations of the course and to prognosticate the clinical illness finally. In this sense, Ab-proteases can be programmed and reprogrammed to suit the requests and standards of regenerative medicine and re-myelination, in particular. This information can allow to design the algorithms for combinatorial (preventive, prophylactic, therapeutic and rehabilitative) treatment, whilst developing unique tools for individually therapy for a number of diseases, such as a group of autoimmune diseases which holds a particular position.

Keywords: Multiple Sclerosis

Abbreviations: PPM: Personalized and Precision Medicine; MS: Multiple Sclerosis; MBP: Anti-Myelin
Basic Protein; SLE: Systemic Lupus Erythematosus; RA: Rheumatoid Arthritis; AH: Acquired Hemophilia; APCs: Ag- 
presenting Cells; AD: Alzheimer's Disease; Tg: Thyroglobulin; ADAPT: Ab-Directed Abzyme Prodrug Therapy.

\section{Introduction}

Today, the society objectively requires a new approach to health care, based on the prevention of diseases, but not on endless and expensive treatment of chronic cases. And those grandiose events that occur today in the world of medical science, once again pay attention to the reconsideration their views on problems related with human health. Thus, an absolutely new model of healthcare service, which includes the principles of Personalized and Precision Medicine (PPM) and integrative medicine and aimed at identifying the disease in early (subclinical) stage, is being created [1].

Meanwhile, to identify the focus of preclinical pathology, it is necessary to create a special system of preclinical criterions and respective predictive diagnostic tests. What tests will allow us to determine with high authenticity the genetic susceptibility to the occurrence of pathologies? First of all, it is genomic testing (genomic screening), which represents the complete picture of the genes and chromosomes of particular patient. This information can allow to design the algorithms of treatment, to create tools for individual tagging therapy for a number of diseases, such as the group of autoimmune diseases holds a unique position. It should be noted, that new targeted therapies for autoimmune and inflammatory diseases would require greater understanding of a patient or a person-at-risk to get the therapy personalized for either of those subsets. Accordingly, now it is time to identify biomarkers of newer generations and to create simultaneously a fundamentally new strategy based upon subclinical recognition of those biomarkers long before the disease clinically manifests itself.

\section{Viewpoint}

For instance, new biomarker-based targeted therapies for autoimmune and inflammatory diseases would require greater understanding of a patient or a person-at-risk to get the therapy personalized for either of those subsets. In this sense, the identification and application of diagnostic, predictive and prognostic biomarkers remain the Holy Grail of modern platforms.

Biomarkers (Figure 1) enable pre-early diagnosis, guide targeted therapy and monitor the activity and therapeutic responses across the diseases. So, biomarker discovery would require a special multi-step, highly complicated but promising strategy to identify, to characterize, to describe, to define the classification niche, to assess the scope of applications and to get them validated.

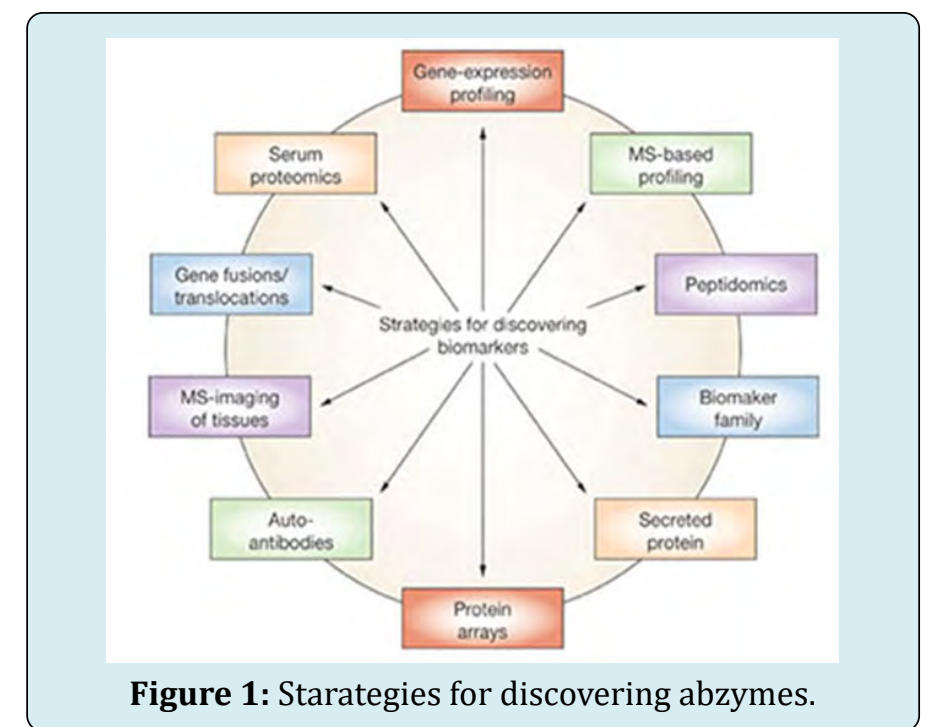

Catalytic antibody (abzyme) is a type of Ab with catalytic activity. The phenomenon of catalysis by auto-Abzs is more and more in research focus. Natural abzymes can be found not only in healthy humans and but also in patients with a broad scope of autoimmune diseases. Studying abzymes can provide important insights into enzyme reaction mechanisms and the immune system itself. In this illustration we summarize new data on abzyme applications in basic science, translational applications, clinical medicine, biotechnology and bioindustry.

Among the best-validated predictive biomarkers are autoimmunity-related ones (including antibodies/Abs) to predict and prognosticate risks of the chronification, complications and thus disabling. The latter is so much valuable and important since chronic autoimmune inflammation course is structured to consist from different stages including subclinical and clinical ones (Figure 2) [2].

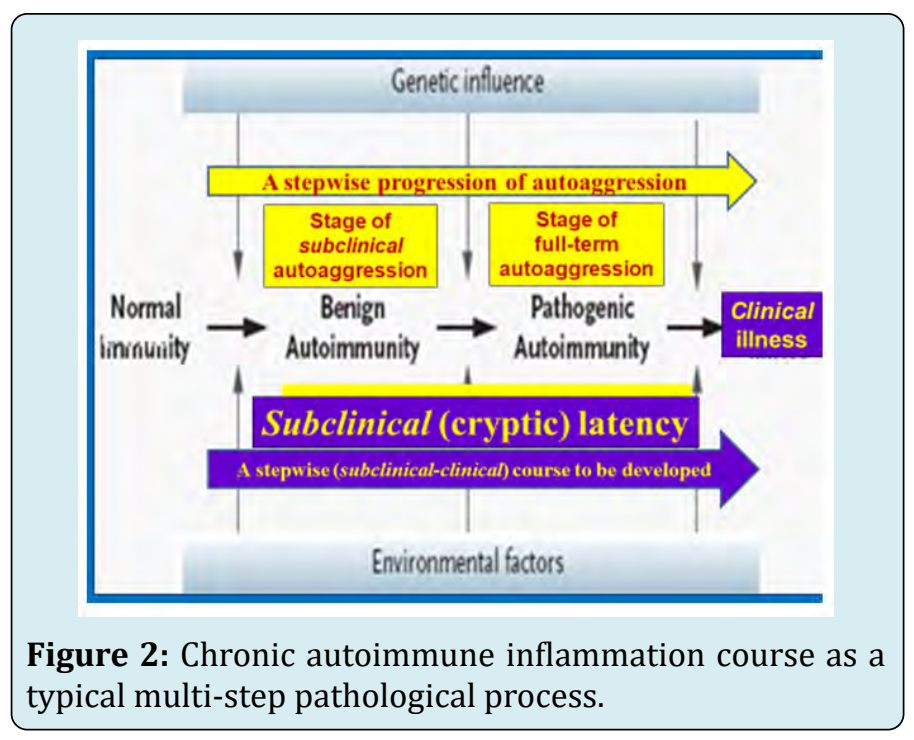


Today, Abs are recognized to elicit an almost unlimited range of reactivities including responses to compounds only recently synthesized in the laboratory and not previously existing in nature. In addition, molecules differing in the smallest detail could be distinguished by their reactivity with different Abs. The highly evolved machinery of the immune system to produce structurally and functionally complex molecules like Abs offers tremendous opportunities for biologists, chemists, bioengineers and practitioners. Whereas the latter provided the framework for understanding the molecular basis of biomolecular structure and function, the immune system provided a highly evolved synthetic and selective process of nature.

Multiple sclerosis (MS) is just one of the chronic tissuespecific autoimmune diseases resulting in a destruction of myelin by different tools, including autoAbs [3]. The crucial step in the pathogenesis of MS is primary myelin damage which is mediated by autoAbs to trigger a release of separate and pathogenically valuable myelin-associated epitopes into the bloodstream. Those epitopes act as sensitizing factors to generate autoAbs (Figure 3).

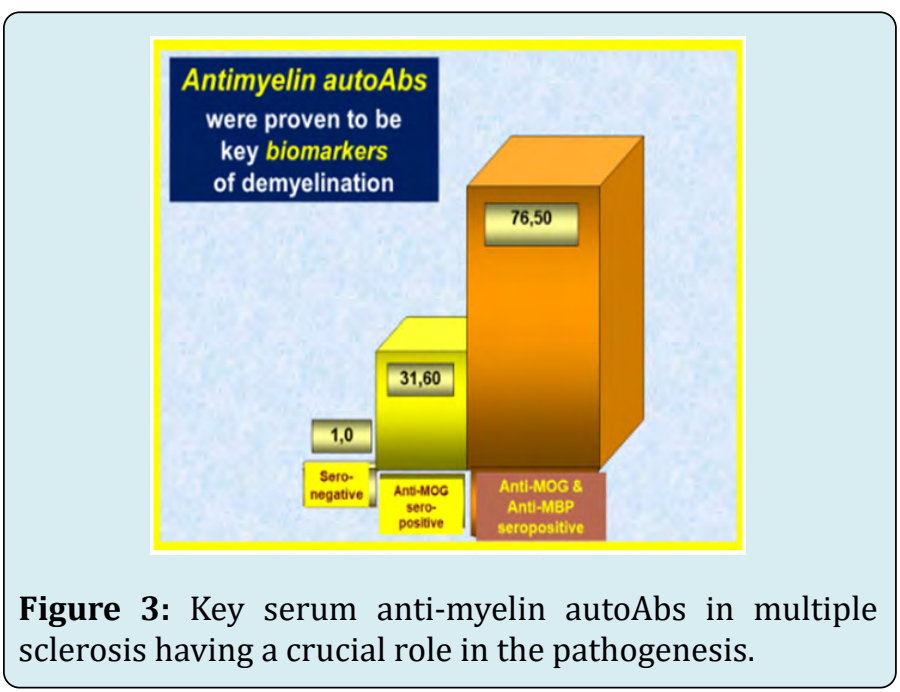

B cells can increase or dampen CNS inflammation, but their proinflammatory effects seem to be more prominent in most patients, as B-cell depletion is a promising therapeutic strategy. Meanwhile, the MS clinical phenotypes, disease courses and responses to treatment that are associated with anti-myelin autoAbs are currently being defined.

Anti-myelin basic protein (MBP) autoAbs have generally been considered to be absent from sera from healthy individuals, but to be detectable in sera from some patients with multiple sclerosis (MS). AutoAb biomarkers are useful in distinguishing subjects with the relapsing-remitting form of MS from those with the secondary progressive subtype.
Myelin basic protein (MBP) is one of the most abundant proteins in CNS. However, its role in MS pathogenesis or prediction of disease progression is still unclear. And antiMBP autoAbs are a marker for MS-associated demyelination and appears to play a significant role in the etiology of multiple sclerosis.

Similar to MBP, the pathogenic role of MOG Abs has been debated intensely, whilst demonstrating their pathogenic potential.

Abs against myelin oligodendrocyte glycoprotein (MOGAbs) are associated with demyelinating syndromes of the CNS. Most patients with MOG-Ab-associated disorders have favourable outcomes, but a subset are left with permanent disability, usually as a result of the initial attack. Many MOGAb-positive MS patients develop relapsing disease; relapses usually involve optic neuritis and often occur during steroid weaning or soon after steroid cessation, suggesting that a longer initial treatment duration is required. Analysis of serum autoAbs against MOG and MBP in patients with a clinically isolated syndrome is a rapid, inexpensive, and precise method for the prediction of early conversion to clinically definite MS. This finding may be important for the counselling and care of patients with a first demyelinating event suggestive of MS. Although, in general, autoAbs against myelin are neither a specific nor a diagnostic feature of MS, it seems that specific demyelinating Abs are involved in the immunopathogenesis of the disorder in at least a subgroup of patients. And the analysis of those Abs can be used to estimate roughly the individual risk of an early first relapse and therefore of clinically definite MS which, in turn, would drive the demyelination and thus the disease progression.

At present, a spectrum of myeline-associated autoAbs occurring in MS patients has been confirmed to be very large. The versatility of Abs is demonstrated by the various functions that they mediate such as neutralization, agglutination, fixation with activation of complement and activation of effector cells. In addition to this plethora of functions, some Abs express enzymatic activity $[4,5]$.

According to classical conception, Abs are specific proteins produced by the immune systems with exclusive function of Ag binding. But Abs against chemically stable analogues modelling the transition states of chemical reaction can catalyze many different reactions and were thus called catalytic Abs or abzymes (derived from $\mathrm{Ab}$ and enzymes), which thus to belong to Abs with a feature of functionality [6].

Abzymes can catalyze more than 200 different chemical reactions and are new biological catalysts attracting great interest during recent years and are well described. 
Catalytic antibodies (CatAbs) are capable of performing almost any type of reaction with high selectivity and stereospecificity and like enzymes process their substrates through a Michaelis complex in which the chemical transformation occurs, followed by product dissociation [7].

"Naturally occurring" CatAbs participate directly in the elimination of the biochemical wastes released by the metabolism of the organism and pointed towards an intrinsic protective role of Abs under physiological conditions. This role is independent of the capacity of Abs to neutralize circulating exogenous Ags, to facilitate their endocytosis by Ag-presenting cells (APCs) and to participate in their elimination from the organism [8].

Abs endowed with enzymatic properties have been described in human autoimmune manifestations for more than a decade in a variety of disorders such as autoimmune thyroiditis, systemic erythematosus (SLE), scleroderma, rheumatoid arthritis (RA), and acquired hemophilia (AH). Abs isolated from these conditions were able to specifically hydrolyze thyroglobulin, DNA, RNA, and factor VIII (FVIII) or factor IX (FIX), respectively. Most of the data accumulated through studies on natural catalytic autoAbs indicate that production scales up markedly in pathological abnormalities. DNA- and RNA-hydrolyzing Abs (DNA and RNA-abzymes) have been isolated from the serum of patients with different systemic autoimmune diseases: systemic lupus erythematosus (SLE), sclerodermia, rheumatoid arthritis (RA) [9].

The first example of an abzyme under pathological conditions in bronchial asthma patients was a case, in which the Abs were able to cleave the vasoactive intestinal peptide (VIP) [10]. Proteolytic Abs specific for thyroglobulin (Tg) have been reported in patients with thyroiditis. Amyloid $b$ peptide (Ab)-hydrolyzing IgM Abs were recently found in the sera of patients with Alzheimer's disease (AD) [11].

The origin of disease-associated CatAbs under pathological conditions is far from clear. Disease-associated CatAbs may have been "induced" by the Ag implicated in the disease. Secondly, the increased occurrence of CatAbs in pathology may result from the loss of repressive control over CatAbs-producing clones generated spontaneously under physiological conditions. A third explanation for the origin of CatAbs in pathological conditions is based on idiotypic network and exacerbated self-recognition in autoimmune diseases.

The CatAbs may complement the general alteration of the immune response. In this respect, the pathological immune response may be directed towards different Ags, some of which are directly relevant to the disease, some of which are not, or alternatively, may be directed against a single Ag that may not be related to the disease. In this sense, proteolytic Abs (Ab-proteases) as the second stage of the discoveries in the area mentioned, would represent Abs to provide proteolytic effects. It is known that proteases precisely control a wide variety of physiological processes and thus are important drug targets.

A protease is a canonical enzyme that performs proteolysis, that is, begins protein catabolism by hydrolysis of the peptide bonds that link amino acids together in a polypeptide chain. Proteases have evolved multiple times, and different classes of protease can perform the same reaction by completely different catalytic mechanisms [8]. So, critical for diverse biological processes, proteases represent one of the largest families of potential and promising and druggable targets.

The precise pathogenesis and etiology of MS as a complex autoimmune disease are still a mystery. Despite many studies that have been aimed to identify biomarkers, no marker has yet been approved for MS. And as it is known, canonical autoAbs play neither predictive nor discriminative role to affect the pre-early and/or subclinical stage of MS. So, there is urgently needed for biomarkers, which could clarify pathology (including subclinical one), monitor disease progression, response to treatment, and prognosis in MS. Immunomic analysis is a set of powerful tools to identify putative and novel candidate biomarkers. Different human compartments analysis using proteomics, cytomics, and bioinformatics approaches has generated new information for further clarification of MS pathology, elucidating the mechanisms of the disease, finding principally new targets, and monitoring treatment response [3]. Overall, omics approaches can develop different therapeutic and diagnostic aspects of MS, from biomarker discovery to PPM.

Briefly! MS is one of the chronic autoimmune inflammatory conditions resulting in a destruction of myelin by different tools, including autoAbs. And a spectrum of myelin-associated autoAbs occurring in MS patients has been confirmed to be very large. What is the "nuclear" in process of disease development?.

The crucial step in the pathogenesis of MS is a primary myelin damage which is mediated by autoAbs to trigger a release of separate and pathogenically valuable myelinassociated epitopes into the bloodstream. Along with canonical Abs, some of the families proven to occur are Abs possessing with catalytic activity (catAbs or abzymes) and thus to belong to Abs with a feature of functionality. As regard CatAbs, it is multivalent Igs, presumably, of IgG isotype, 
endowed with a capacity to hydrolyze the Ag substrate. The property mentioned is buried in the Fab-fragment of the Ig molecule and is appearing to sound as a functional property of the $\mathrm{Ab}$ molecule. In this sense, proteolytic Abs (or Ab-proteases) as a significant portion of the big family of abzymes represent Abs endowed with a capacity to provide targeted proteolytic effect.

Abs against myelin basic protein/MBP with proteolytic activity exhibiting sequence-specific cleavage of MBP are of great value to monitor demyelination whilst in MS. The activity of Ab-proteases was first registered at the subclinical stages 1-2 years prior to the clinical illness. And the activity of the Ab-proteases revealed significant correlation with scales of demyelination and the disability of the patients as well (Figures 4A-4C) [4].
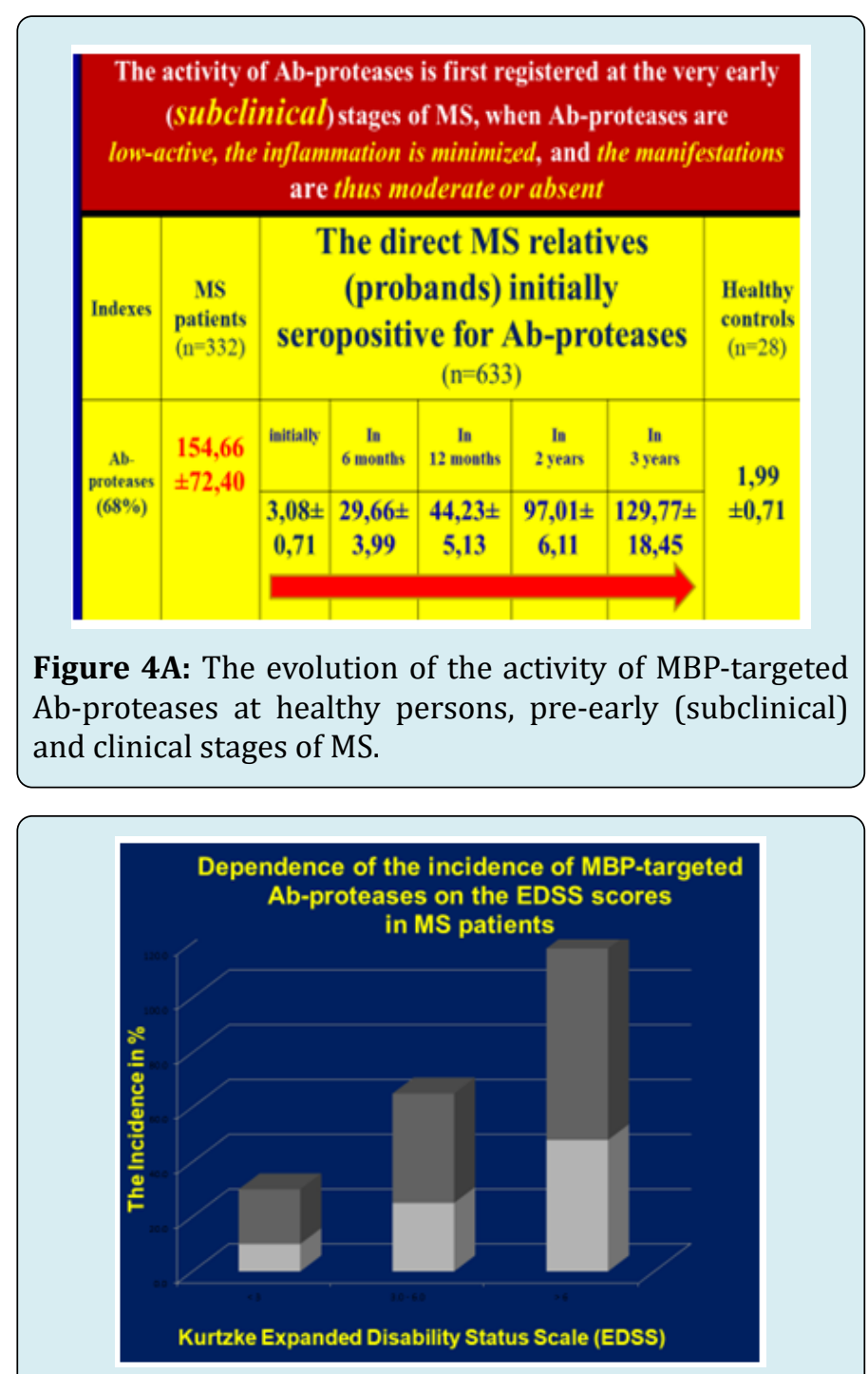

Figure 4B: Dependence of the incidence of MBP-targeted Ab-proteases on the EDSS score in MS patients.

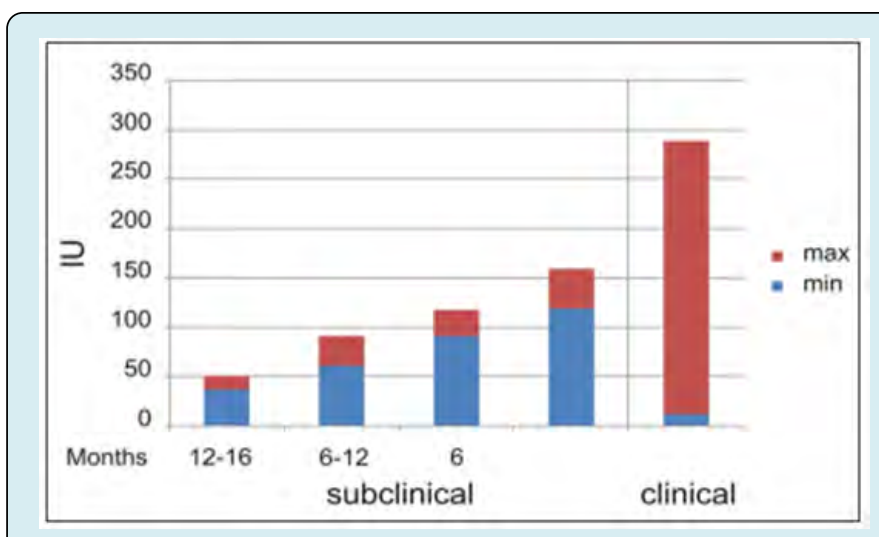

Figure 4C: The evolution of the activity of MBP-targeted Ab-proteases at subclinical and clinical stages of MS.

Sequence-specific Ab-proteases have proved to be greatly informative and thus valuable as biomarkers to monitor MS at both subclinical and clinical stages! Therefore, the proposed predictive value of MBP-targeted Ab-proteases for the development of MS is being challenged! So, the activity of $\mathrm{Ab}$-proteases and its dynamics tested would confirm a high subclinical and predictive value of the tools as applicable for monitoring protocols [12].

Of tremendous value are $\mathrm{Ab}$-proteases directly affecting remodeling of tissues with multilevel architectonics (for instance, myelin). By changing sequence specificity one may reach reduction of a density of the negative proteolytic effects within the myelin sheath and thus minimizing scales of demyelination.

Targeted Ab-mediated proteolysis could be also applied to isolate from Ig molecules catalytic domains directed against encephalitogenic autoepitopes or domains containing segments to exert proteolytic activity. So, further studies on Ab-mediated MBP degradation and other targeted $\mathrm{Ab}$-mediated proteolysis may provide biomarkers of new generations and thus a supplementary tool for assessing the disease progression and predicting disability of the patients and persons-at-risks.

To achieve the implementation of PPM concept into the daily practice, it is necessary to create a new strategy based upon recognition, testing and monitoring of biomarkers of the newest generation. In this sense, CatAbs (abzyme) is a type of Abs with catalytic activity being found not only in healthy humans and but also in patients with autoimmune diseases. Studying abzymes can provide important insights into enzyme reaction mechanisms and the immune system itself. 


\section{Discussion}

What does arise in response to «a call» of this kind of information received? Naturally, it is the emergence of nanotechnology, specifically, the design of new diagnostic tools and new targeted therapeutics based on principles of artificial biocatalysts and drug design. The traditional goal of $\mathrm{Ab}$ engineering is to combine various $\mathrm{Ab}$ domains to generate customized Abs that show specialized binding properties, optimal half-lives and desirable effector functions. Abs can be engineered to make proteins of higher affinity or smaller molecular variants that retain or change the functional properties of the original $\mathrm{Ab}$. So, biomarkers and integration of diagnostics with therapeutics are becoming important for the selection and monitoring of individualized treatments [13].

The pathogenic or beneficial effect of ABZYMES has never been directly demonstrated by reproducing the disease in animals through passive administration of CatAbs isolated from patients' serum. The translational potential of $\mathrm{Ab}$ proteases and the knowledge is in the rational design of new diagnostic tools and new therapeutics based on principles of artificial biocatalysts and biodesign.

The traditional goal of $\mathrm{AB}$ engineering is to combine various $\mathrm{Ab}$ domains to generate customized Abs that show specialized binding properties, optimal half-lives and desirable effector functions. Abs can be engineered to make proteins of higher affinity or smaller molecular variants that retain or change the functional properties of the original $\mathrm{Ab}$ [14].

Meanwhile, Ab-proteases can be programmed and reprogrammed to suit the needs of the body metabolism or could be designed for the development of new catalysts with no natural counterparts.

And the next important step in the direction of the innovations-based approach should be their early adoption in clinics for future medical interventions! In this sense, neurodegenerative diseases are promisingly suited models for PPM because of the rapidly expanding Hi-Tech innovations and translational resources including ABZYMES technologies and the development of biomarkers and the potential modifying treatments and Personalized Precision Neurology (PPN) is the application of principles of PPM.

Several biotechnologies are being integrated to develop PPM and PPN. Besides BASICS, postgenomic technologies such as nanoimmunotechnology are also used. So, biomarkers and integration of diagnostics with therapeutics are becoming important for the selection and monitoring of individualized treatments to get neurodegenerative disorders cured. And accurate and precise prediction is crucially vital for prevention of autoaggression, and the targeted preventive, therapeutic and rehabilitative treatment could thus be given to those individuals who are most likely to develop the disease [15].

The application of the newest and upgraded translational applications to the treatment and prevention of neurodegenerative disorders appear to be highly promising in contrast to the traditional "one-drug-fits-all" approach. And Ab-based therapeutics are entering the key stage of drug discovery (Figure 5).

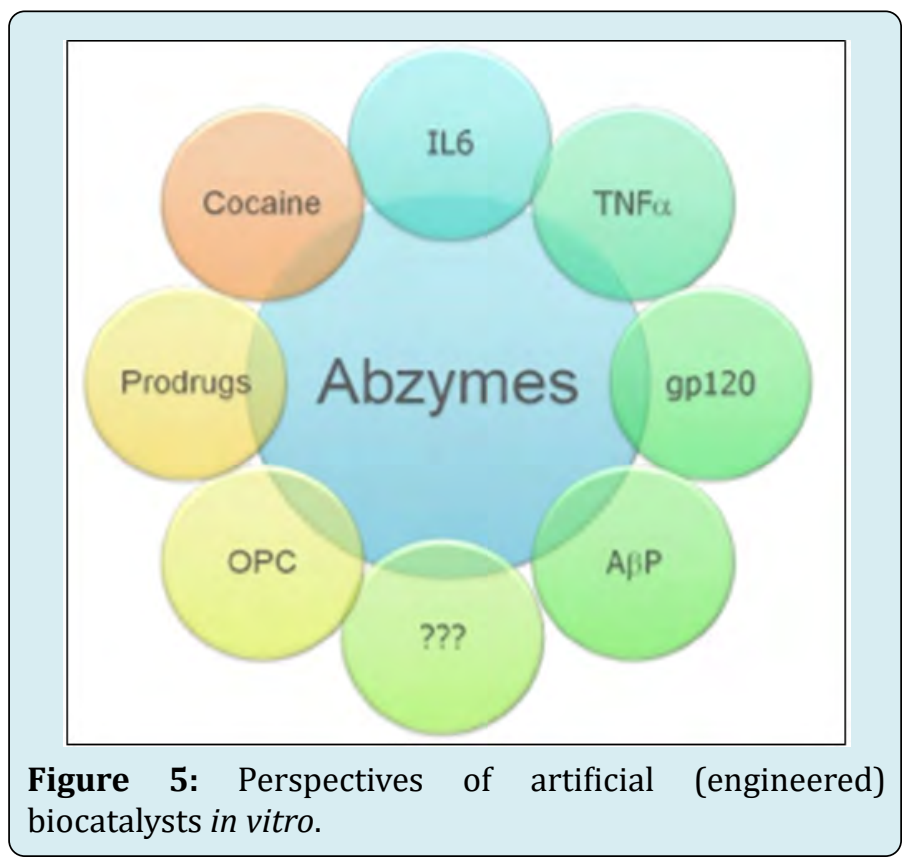

IL, interleukin; TNF, tumor necrosis factor; gp120, a glycoprotein exposed on the surface of the HIV envelope. $\mathrm{OPC}$, oligodendrocyte precursor (progenitor) cell; $\mathrm{A} \beta \mathrm{P}$, antiamyloid beta protein Abs [16].

As a result of a major shift in focus of many biopharma companies. For instance, prodrug activation by CatAbs conjugated with targeted Abs, called Ab-directed abzyme prodrug therapy (ADAPT), might be proposed as a strategy for site-specific drug delivery systems for myelin degradation preventing drugs. To achieve ADAPT, we should focuse on specific requirements for prodrugs and CatAbs, the stability of the prodrugs against natural enzymes, and the applicability of abzymes for a wide range of prodrugs. As an example, you might consider CatAbs generated by immunization of a vitamin B6 phosphonate transition state analog. The elicited Abs were found to hydrolyze several anti-inflammatory prodrugs with the vitamin B6 pro-moiety on one hand, and to secure Ab-catalyzed prodrug. So, the combination of CatAbs and prodrugs masked with the targeted bioactive 
ingredients would allow the targeted drugs to be used [17].

The other example is artificial CatAbs based on welldefined metal clusters modified with rationally designed peptides, termed clusterbodies, which possess favorable integrated features of matched ultrasmall sizes, intrinsic fluorescence, and enzyme-like catalytic and selective recognition properties that are inaccessible to traditional Abs. Consequently, a quantitative assay with high accuracy and high sensitivity is established by measuring the fluorescence and catalytic chemiluminescence of metal clusters preferentially recognizing the protein biomarker (including targeted Ags or Abs), which is confirmed by the molecular-weight marker references of immunoblotting. This platform has potential application in analyzing lowabundance protein biomarkers (including targeted Ags and Abs) in complex biological matrixes, which is essential to autoimmunity conditions in early and pre-early stages. It inspires the construction of clusterbody-based precise bioprobes with customized structures and integrative functions for advanced quantitative biosensing $[18,19]$. In this context, the development in neurology of a PPM approach could represent an excellent possibility to identify subclinical stages of disease, make adequate differential diagnosis and provide timely and optimal treatments instead of the traditional treatments which are utilized at later stage of disease. With advances in our understanding of Ab-protease functions and properties coupled with improvements in immune and protein engineering, we can expect that $\mathrm{Ab}$-protease therapeutics will gain regulatory approval and make significant contributions in healthcare in the near-term.

Utilizing globally PPM in MS requires 3 crucial components:

- Assessment of prognosis soon after diagnosis;

- Considering an early therapeutic plan based on risk benefit and patient predilections;

- A pre-early assessment of response to therapy and taking alternative therapies in the case of failure.

And thus biomarkers are important for understanding MS patient disease profile, prognosis, diagnosis, and disease course prediction. They are also critical in identifying the benefits and side effects of new therapies on patients (therapy-oriented) and/or persons-at-risk (preventively oriented).

We would stress that the future biomarkers' discovery and execution will face numerous challenges. And consequently, serious cooperative attempts among biodesigners, translational researchers, clinicians, and bioindustry are needed. This collaboration will move us one step forward from hope to the ultimate goal of PPM in MS. We hope that standardizing critical elements of proteomics studies, as well as immunomics signature would provide insights into the pathogenesis of MS and valuable clinical and translational applications shortly.

\section{Conclusion}

We are experiencing a Renaissance primarily driven by next-generation biotechnologies. In this sense, neurodegenerative diseases are promisingly suited models for PPM because of the rapidly expanding Hi-Tech innovations and translational resources including ABZYMES Technologies and the development of biomarkers and the potential modifying treatments. In this context, the development in neurology of a PPM approach could represent an excellent possibility to identify subclinical stages of disease, make adequate differential diagnosis and provide timely and optimal treatments instead of the traditional treatments which are utilized at later stage of disease [20].

Further studies on Ab-mediated MBP degradation and other targeted Ab-mediated proteolysis may provide biomarkers of the next step generations and thus supplementary tools for assessing the disease progression and predicting disability of the patients and persons-atrisks. Those tools are needed to secure artificial or edited $\mathrm{Ab}$-proteases as unique translational probes to diagnose, to monitor, to control and to treat and rehabilitate MS patients at clinical stages and to prevent the disorder at subclinical stages in persons-at-risks to secure the efficacy of regenerative manipulations.

\section{References}

1. Bodrova TA, Kostyushev DS, Antonova EN, Slavin S, Gnatenko DA, et al. (2012) Introduction into PPPM as a new paradigm of public health service: an integrative view. EPMA J 3(16): 16.

2. Alexander G Gabibov, Natalya A Ponomarenko, Eugenia B Tretyak, Mikhail A Paltsev, Sergey V Suchkov (2006) Catalytic autoantibodies in clinical autoimmunity and modern medicine. Auto Immune Rev 5(5): 324-330.

3. Ponomarenko NA, Durova OM, Vorobiev II, Belogurov AA Jr, Kurkova IN, et al. (2006) Autoantibodies to myelin basic protein catalyze site-specific degradation of their antigen. Proc Natl Acad Sci U S A 103(2): 281-286.

4. Ponomarenko NA, Durova OM, Vorobiev II, Belogurov AA, Telegin GB, et al. (2006) Catalytic activity of autoantibodies toward myelin basic protein correlates with the scores on the multiple sclerosis expanded disability status scale. Immunol Lett 103(1): 45-50.

5. Weber MS, Hemmer B, Cepok S (2011) The role of 
antibodies in multiple sclerosis. Biochim Biophys Acta 1812(2): 239-245.

6. Tramontano A, Janda KD, Lerner RA (1986) Catalytic antibodies. Science 234(4783): 1566-1570.

7. Pollack SJ, Jacobs JW, Schultz PG (1986) Selective chemical catalysis by an antibody. Science 234(4783): 1570-1573.

8. Friboulet A, Avalle B, Debat H, Thomas D (1999) A possible role of catalytic antibodies in metabolism. Immunol Today 20(10): 474-475.

9. Shuster AM, Gololobov GV, Kvashuk OA, Bogomolova AE, Smirnov IV, etal. (1992) DNA hydrolyzing autoantibodies. Science 256(5057): 665-667.

10. Paul S, Volle DJ, Beach CM, Johnson DR, Powell MJ, et al. (1989) Catalytic hydrolysis of vasoactive intestinal peptide by human autoantibody. Science 244(4909): 1158-1162.

11. Wootlaa B, Desmazesc SL, Warringtona AE, Biebera AJ, Kaveric SV, et al.(2011) Autoantibodies with Enzymatic Properties in Human Autoimmune Diseases. J Autoimmun 37(2): 144-150.

12. Xu Y, Yamamoto N, Janda KD (2004) Catalytic antibodies: hapten design strategies and screening methods. Bioorg Med Chem 12(20): 5247-5268.
13. Lefèvre SP, Naya RB, Shahsavarian MA, Friboulet A, Avalle B (2014) Catalytic antibodies and their applications in biotechnology: state of the art. Biotechnol Lett 36(7): 1369-1379.

14. Rao DN, Wootla B (2007) Catalytic antibodies: Concept and promise. Resonance 12: 6-21.

15. Diasab S, Jovicac F, Renard PY, Taranc F, Créminona C, et al. (2002) Immunologically driven chemical engineering of antibodies for catalytic activity. J Immunol Methods 269(1-2): 81-98.

16. Taguchi H, Planque S, Nishiyama Y, Symersky J, Boivin $S$, et al. (2008) Autoantibody-catalyzed hydrolysis of amyloid beta peptide. J Biol Chem 283(8): 4714-4722.

17. Dimiter S Dimitrov (2019) From Catalytic Antibodies to Antibody-Drug Conjugates. Cell Chemical Biology 26(9): 1200-1201.

18. Motherwell WB, Bingham MJ, Six Y (2001) Recent progress in the design and synthesis of artificial enzymes. Tetrahedron 57(22): 4663-4686.

19. Mayforth RD, Quintáns J (1990) Designer and Catalytic Antibodies. N Engl J Med 323: 173-178.

20. Suchkov SV, Alekberova ZS, Paleev FN, Naumova TE, Misikov VK, et al. (2005) Achievements and prospects of clinical abzymology. Vestn Ross Akd Med Nauk 9: 38-43. 